\title{
VenDASys - A Versatile Experimentation Platform for Educational Purposes
}

\author{
doi:10.3991/ijoe.v6i3.1350 \\ R. Sallier ${ }^{1}$, R. Mißler ${ }^{1}$, B. Brück ${ }^{2}$ and A. Schütze ${ }^{1}$ \\ ${ }^{1}$ Saarland University, Saarbrücken, Germany \\ ${ }^{2}$ Max Planck Gymnasium, Saarlouis, Germany
}

\begin{abstract}
In this paper we present an approach to increase the interest of pupils and high school students in modern science and technology. To achieve this, we developed a versatile experimentation platform for the use in schools and school projects, called VenDASys (Versatile Control and Data Acquisition System). VenDASys allows the interfacing of individual (micro-)sensors or complete experimental set ups while minimizing the required electronics, which are often frustrating for students and teachers alike due to limited experience in this field. Thus, students (and teachers) can directly concentrate on and successfully deal with different sensors and experiments based on these sensors. We present the concept with an example project on blood pressure monitoring. Getting involved in comprehensible MEMS (Micro Electro Mechanical Systems)-applications and solvable problems at a relatively early stage of their education will definitely increase the chances that high school students decide to choose a technical education later.
\end{abstract}

Index Terms-open experimentation platform; microsensors

\section{INTRODUCTION AND MOTIVATION}

In the last years the gap between the need and the availability of qualified personnel has been increasing in many technical and scientific fields. One direct consequence is the current drastic shortage of engineers. This problematic situation has been addressed in many activities around the world in didactics and education in different ways, e.g. [1, 2]. The MEMS industry in Germany already regards this issue as a major challenge for future economic success. For this reason, in 2002, the German Federal Ministry for Research and Education (BMBF, Bundesministerium für Bildung und Forschung) initiated the foundation of six networks focusing on MEMS education and training [3]. One of the major goals of these networks, and especially for the network "pro-mst" [4], was to counteract this trend by increasing the interest of high school students in natural sciences and engineering.

Our learning lab SinnTec (Sinn für Technik - Technische Sinnesorgane) was founded as result of our experiences within the network "pro-mst". SinnTec addresses both high school students and teachers to gain hands-on experience. One of the major topics of this lab is to show where MEMS technology is used today, e.g. in a car. Secondly, students should actively work with microsensors as technical senses to gain a deeper understanding. From these activities [5] we learned that difficulties in working with sensors do not primarily arise from the basic understanding of sensor function principles or MEMS

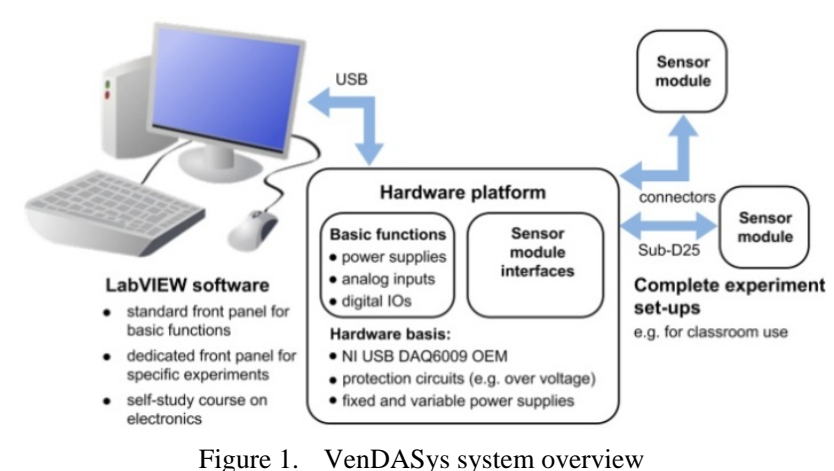

production processes but from the electronics required for sensor read out and its useful application.

Based on this experience, we developed a universal and modular experimentation platform for use in schools and school projects with an open source concept allowing exchange of experiments between schools, teachers and students [6]. The VenDASys platform offers a very comfortable and straightforward opportunity to deal with sensors by simply "bypassing" most of the electronics from a didactical point of view. Thus students and teachers in a simple way gain access to the field of MEMS in particular and science and technology in general by realizing their own ideas for experiments.

\section{TECHNICAL CONCEPT}

The VenDASys consists of three main parts (Fig. 1): (a) a hardware platform providing basic functionalities like power supplies, measurement inputs and digital IOs plus dedicated sensor interfaces, (b) a multi-purpose software tool with graphical user interface (GUI) implemented in LabVIEW, NI, and (c) dedicated sensor modules for different applications. The hardware platform is connected to a PC via an USB interface; individual sensors are connected to the platform via standard lab-cables, especially during the development phase. When application specific sensor modules have been realized, they can quickly be connected via dedicated Sub-D25-interfaces with power supply, analog and digital in- and output as well as data exchange. The LabVIEW-GUI gives the user full access to a variety of hardware functions (mainly analog and digital in- and outputs) without having to address the hardware set up in detail. A more detailed description is given below.

\section{A. Hardware platform}

The electronic hardware of the system consists of several modules, with the core of the VenDASys being an 
USB DAQ 6009 OEM module, a commercial data acquisition system manufactured by NI. To prevent damaging the module during the course of development work by inexperienced high school students, we added additional protection circuits, i.e. against overvoltage, short circuiting or polarity reversal. The main task of the DAQ module is the acquisition of analog and digital inputs from sensors, switches etc. Furthermore, the DAQ controls the digital outputs of the system which can be used for control purposes as well as variable voltage and current sources which can again be used for control purposes or to supply external circuits. Further modules of the VenDASys are constant voltage sources (for supplying external electrical circuits), relay circuits (for switching periphery requiring higher power) and finally a separately oscilloscope with an integrated signal generator. Table I gives an overview of the integrated hardware functions.

By connecting sensors and other elements to the hardware platform, the need for separate power supplies, instruments etc. is obviated. The VenDASys platform therefore performs all the functions of a small electronics laboratory. For the acceptance by students and teachers alike, the proper, robust and transparent function is of utmost importance. We have therefore balanced the complexity of system, i.e. the number of in- and outputs, with the simplicity of use. For this, the module interfaces are especially important as they allow setting up complete experiments with just one connection. Fig. 2 shows the frontpanel of the hardware platform with the different features. The DAQ module and the separate oscilloscope come with LabVIEW software tools allowing an easy handling of the front-panel functionalities when connected to a PC via USB.

\section{B. Software tool}

The PC software is implemented in LabVIEW because of its wide spread use in measurement applications. LabVIEW is a graphical programming language developed and optimized for the use in measurement and automation, which is divided in two basic parts: the block diagram and the front panel. The block diagram represents the programmer's working area while the front panel (example shown in Fig. 3) acts as the GUI of the completed program. The benefit for students and teachers is, that minimal experience with the programming language is required to develop programs with attractive optics for control and data display, as standard elements from the LabVIEW toolbox can be used to realize the required functions in short time.

To further simplify the development of programs for specific experiments a Virtual Instrument (VI) was realized, i.e. a single element or function in LabVIEW providing access to the complete functionality of the VenDASys hardware platform. By integrating this element in their own programs the complete communication and internal operation of the hardware/software combination is hidden for students. In this way, they can concentrate on the experiment itself without distraction from the electronics or the data communication. Give all authors' names; use "et al.” if there are six authors or more. Papers that have not been published, even if they have been submitted for publication, should be cited as "unpublished" [4]. Papers that have been accepted for publication should be cited as "in press” [5]. In a paper title, capitalize the first word and all
TABLE I.

OVERVIEW OF VENDASYS HARDWARE FEATURES

\begin{tabular}{|c|c|}
\hline Features & Detailed properties \\
\hline $\begin{array}{l}5 \text { power } \\
\text { supplies }\end{array}$ & $\begin{array}{l}\text { - constant voltage source }+5 \mathrm{~V} /+1 \mathrm{~A} \\
\text { - constant voltage source }+11 \mathrm{~V} /+1 \mathrm{~A} \\
\text { - constant voltage source }-11 \mathrm{~V} /-1 \mathrm{~A} \\
\text { - } 2 \text { variable voltage sources } \pm 11 \mathrm{~V} \text { or } \\
2 \text { variable current sources }[0-100] \mu \mathrm{A},[0.2-1] \mathrm{A}\end{array}$ \\
\hline $\begin{array}{l}8 \text { analog } \\
\text { inputs }\end{array}$ & $\begin{array}{l}\bullet \pm 10 \mathrm{~V} \text { range, } 14 \text { bit resolution } \\
\bullet 8 \text { channels for absolute voltage measurement } \\
\text { or } 4 \text { channels differential voltage measurement } \\
\bullet \text { overvoltage protection }\end{array}$ \\
\hline oscilloscope & $\begin{array}{l}\text { - } 250 \mathrm{kHz}, 2 \mathrm{MSamples} / \mathrm{s} \\
\text { - } 2 \text { channels } \\
\text { • integrated signal generator }\end{array}$ \\
\hline $\begin{array}{l}12 \text { digital } \\
\text { in-/outputs }\end{array}$ & $\begin{array}{l}\text { - TTL level } \\
\text { • high impedance input } \\
\text { • over voltage protection } \\
\end{array}$ \\
\hline 2 relay circuits & $\begin{array}{l}\text { - maximum voltage 250VAC/60VDC } \\
\text { - maximum current } 15 \mathrm{~A}\end{array}$ \\
\hline $\begin{array}{l}2 \text { sensor mo- } \\
\text { dule interfaces }\end{array}$ & $\begin{array}{l}\text { - 25-pin sub-D interface mapping all front-panel } \\
\text { functions }\end{array}$ \\
\hline
\end{tabular}

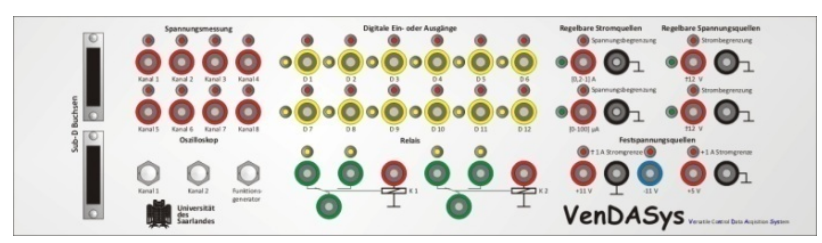

Figure 2. Front panel of the hardware platform

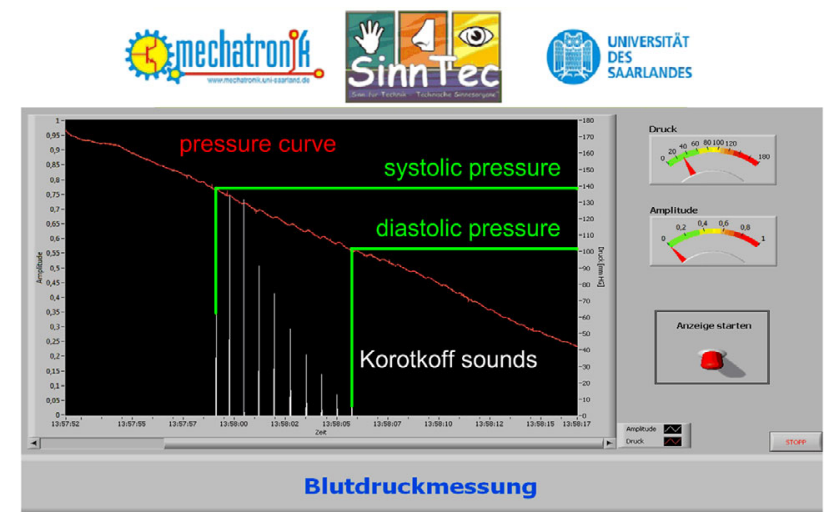

Figure 3. Example of a front-panel in LabVIEW, here showing the signals from pressure sensor and microphone for the blood pressure measurement (see below).

other words except for conjunctions, prepositions less than seven letters, and prepositional phrases.

\section{Sensor modules}

The system offers two options to connect the sensors with the VenDASys in order to set-up complex experiments. The first option uses standard lab-cables, which are connected individually for the different elements in the experiment. This option is used during the development phase of experiments enabling flexible connections and fast adaptation when errors are made. The second option is the connection of complete sensor modules or even experiments via Sub-D25-connectors, which is used after the development phase is complete in order to provide a fast and correct set-up of experiments. This option is especially useful for classroom experiments, when there is 
little time for setting up and high dependability is required.

\section{BloOd Pressure Monitoring: An ExAMPLE}

To illustrate the use of VenDASys for experiments with students, we will describe a new experiment that was recently realized to convey fundamental knowledge on pressure sensors as well as outlining an everyday application. After discussing the different uses of pressure sensors, e.g. for weather stations or height measurements, to monitor tire pressure etc, we chose a medical application in order to point out that VenDASys cannot only be used in physics and technology but also for other natural sciences like biology and chemistry.

The technical background of the experiment is the blood pressure (BP) measurement based on the widely used concept of Riva-Rocci as depicted in Fig. 4. A cuff is used to apply pressure on the arteries while the physician listens to the so called Korotkoff sounds in the brachial artery at the elbow using a stethoscope. In the experiment a pressure sensor is used for the BP measurement and a microphone - essentially another pressure sensor for dynamic pressure changes - to record the Korotkoff sounds. This emulates the function principle of comercial automatic blood pressure monitors for home use.

In addition, the experiment addresses the function principle of the pressure sensor itself and the necessity to calibrate sensors before use. In order to allow a larger group of students (up to 16) to work, we divided the experiment into four sections or experimental stations as shown in Fig. 5. After explaining the overall goal of the experiments and some background on blood pressure measurement, the first two stations address necessary basic electronics and sensor concepts, i.e. a Wheatstone bridge circuit for evaluating resistive sensors and strain gauges for force, weight or pressure. The third station addresses the calibration of the pressure sensor, while in the fourth and final stage an actual blood pressure measurement is performed using the previously calibrated sensor. All stations make use of the principle of activitybased teaching, so that students have a real hands-on experience and do not only watch a teacher setting up and performing a demonstration experiment. Of course, the necessary equipment is prepared and the students are given a handbook guiding them through the experiment.

In the first three stations, basic VenDASys functions, i.e. power supply and analog measurement, are used; for the final station a dedicated program for recording and displaying the measured data and determining the blood pressure is used (Fig. 3) exemplifying the broad VenDASys spectrum.

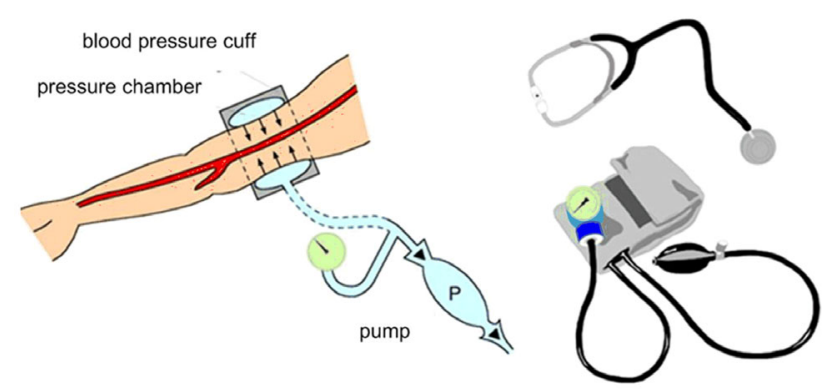

Figure 4. Blood pressure measurement according to Riva-Rocci [7]
Station 1

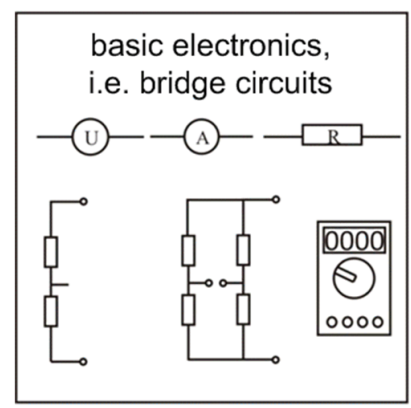

Station 3
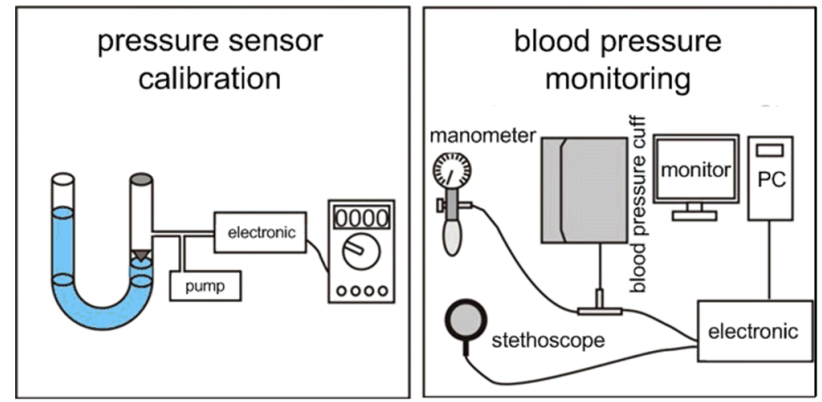

Figure 5. Four experimental stations of the BP monitoring experiment.

\section{A. Theoretical background}

Historically arterial pressure was measured using the height of a column of mercury to reflect the circulating pressure. Today blood pressure (BP) values are still reported in millimeters of mercury (mmHg), even though modern instruments no longer use mercury. For each heartbeat, BP varies between systolic and diastolic pressures. Systolic pressure is the peak pressure in the arteries occurring near the end of the cardiac cycle when the ventricles are contracting. Diastolic pressure is the minimum pressure in the arteries occurring near the beginning of the cardiac cycle when the ventricles are filled with blood. An example of normal measured values for a resting, healthy adult human is $115 \mathrm{mmHg}$ (approx. $15.3 \mathrm{kPa}$ ) systolic and $75 \mathrm{mmHg}$ (approx. $10 \mathrm{kPa}$ ) diastolic (written as 115/75 $\mathrm{mmHg}$, and spoken as "one-fifteen over seventy-five") [8].

The most common method for BP monitoring is the indirect measurement according to Riva-Rocci (Fig. 5.), also called the auscultatory method (from the Latin for listening). In this method, a pressure greater than the systolic pressure is applied using a cuff which collapses the artery. Thus, the blood pressure wave cannot continue in the direction of the hand and no pulse can be felt or heard in the lower arm. The cuff pressure is then slowly reduced and the Korotkoff sounds are recorded. When the pressure falls below the systolic pressure, i.e. when blood just starts to flow in the artery, the turbulent flow creates a "whooshing" or pounding (first Korotkoff sound). The pressure at which this sound is first heard or recorded is the systolic $\mathrm{BP}$. The cuff pressure is further released until the artery is open permanently, i.e. no more sound is heard (fifth Korotkoff sound), at the diastolic arterial pressure [8]. The process is depicted in Fig. 6. The Korotkoff sounds are either manually registered using a stethoscope or automatically using a microphone. 
Thus, for BP monitoring two quantities have to be measured using appropriate sensors: the static pressure in the cuff and the Korotkoff sounds in the brachial artery at the elbow. As sound is basically a high frequency pressure fluctuation, both sensors are very similar. The experiment concentrates on the static pressure sensor which is easier to understand, but also requires higher precision. A standard microphone with audio amplifier is used for recording the Korotkoff sounds.

\section{B. Pressure sensor function principle and calibration}

Pressure sensors today are commonly manufactured using microtechnologies; they were one of the first mass applications of MEMS. The sensor basically consists of a Si chip with thin membrane and integrated piezo-resistive strain gauges in a bridge configuration (Fig. 7). A pressure difference across the membrane leads to a bending of the membrane and thus an electrical signal. With a vacuum on one side of the membrane by applying a glass cap the absolute pressure can be measured.

Conversion of the mechanical quantity pressure, i.e. force per area, into an electrical quantity requires several steps. In the first step the pressure is converted into surface tension by the membrane, on which surface zones

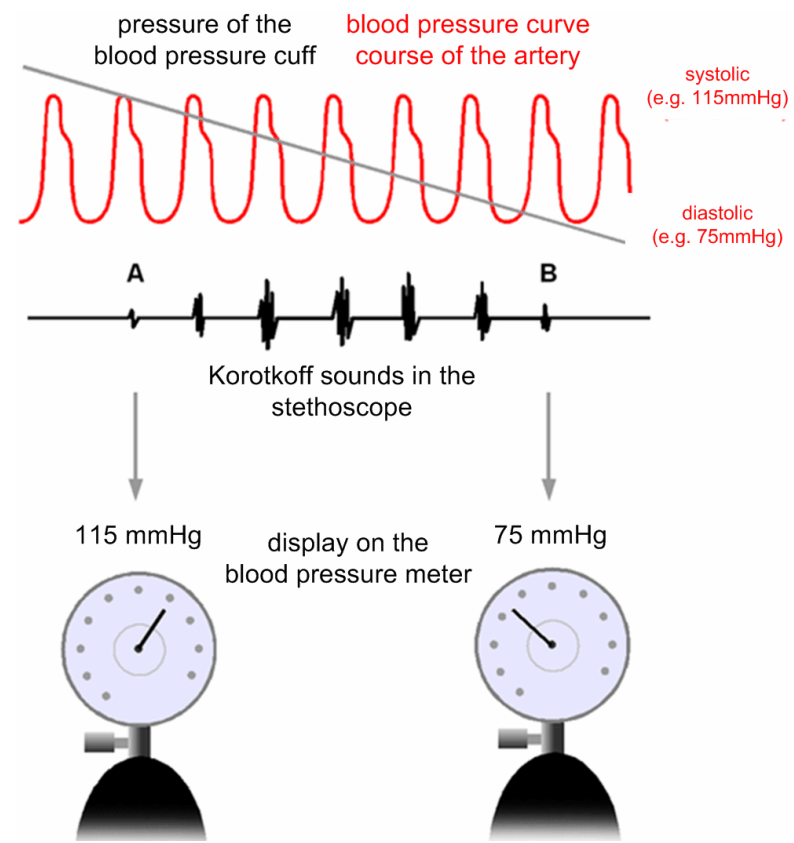

Figure 6. Blood pressure measurement based on Korotkoff sounds [9]

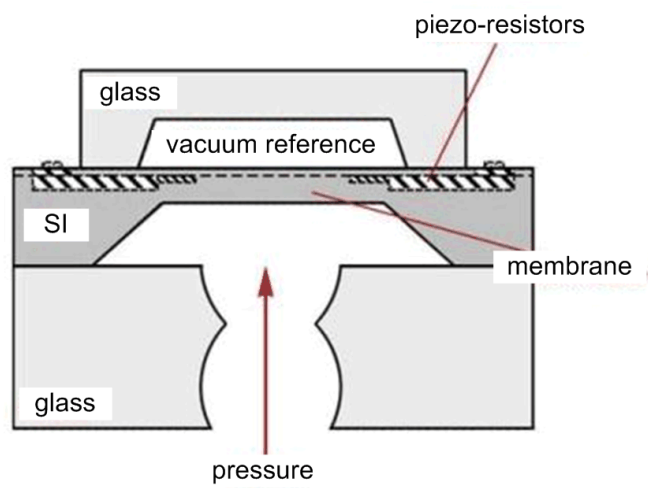

Figure 7. Schematic of a piezo-resistive absolute pressure microsensor [10] will exhibit tensile and compressive strain. Piezo resistors convert this strain into resistance changes, which is finally converted to a voltage by the bridge configuration. Since the strain and the resistance changes are small, the output voltage is also small and has to be amplified for recording. Thus, in the experiment stations the function principle of a Wheatstone bridge and strain gauges are addressed as necessary fundamentals for the pressure sensor. To make the pressure sensor, which is only a few $\mathrm{mm}$ square in size, more accessible to students we developed a macro model which is used to explain the sensor function principle, Fig. 8.

After explaining the function principle students work with a Wheatstone bridge and strain gauges for a weight measurement in the first two experimental stations, respectively. In the third station, a pressure sensor is calibrated using a water (instead of Mercury) column, Fig. 9. The sensitivity of the sensor (in $\mathrm{mV} / \mathrm{mmHg}$ ) is calculated from the measurements of height vs. output voltage and the densities for water and mercury.

\section{BP monitoring with the VenDASys experiment}

After addressing the scientific and technical fundamentals in the introductory talk and the first three stations

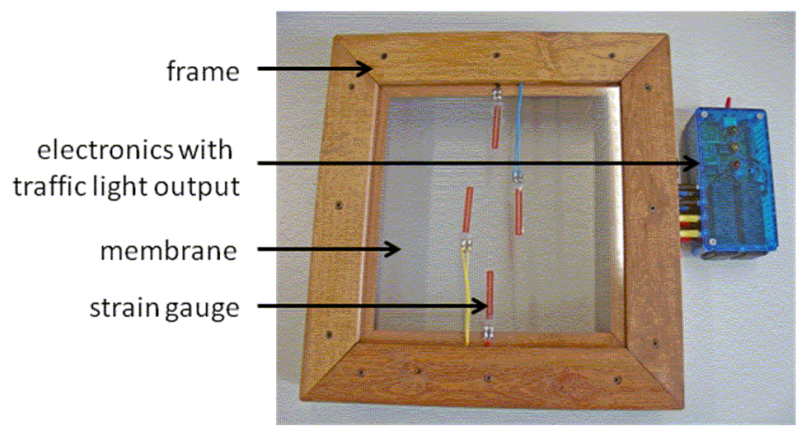

Figure 8. Macro model of a pressure sensor (size approx. $30 \mathrm{~cm}$ square). Applying pressure to the membrane results in a simple output (green: low, yellow medium and red high pressure, respectively).

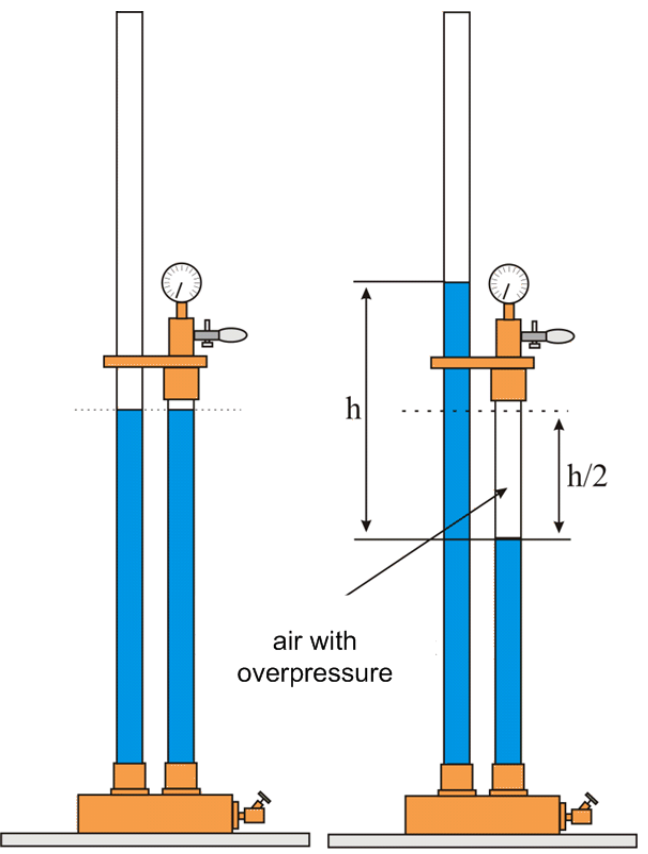

Figure 9. Calibration of the pressure sensor using a water column 
students use a designated VenDASys experiment set-up for monitoring their own blood pressure in the final station. At the beginning of the experiment the necessary components are introduced and explained. Fig. 10 shows the microphone with amplifier, the blood pressure cuff with manual pump as parts of the complete experimental set-up. The experiment is set-up by the students themselves based on an instruction guide.

Then, students can either use existing software to run the experiment or, if more time is available, realize their own program for recording and displaying sensor data and extracting the correct blood pressure values using pressure sensitivity obtained by the calibration in station 3. Finally, students take their own blood pressure using the set-up as shown in Fig. 11.

Fig. 3 shows the front panel of the standard LabVIEW software for BP measurement. In the main window, the pressure curve (red) and the signal intensity recorded by the microphone (white) are displayed; in addition analog scales (top right) show the current sensor values. After pressurizing the cuff until no noise is recorded (no reading on the appropriate scale) data recording is started with the button on the bottom right. When the pressure in the cuff is slowly reduced, the diminishing pressure and the Korotkoff sounds are recorded and displayed. From this diagram the systolic (onset of Korotkoff sounds) and diastolic (disappearance of Korotkoff sounds) pressures can be extracted as shown in Fig. 3.

With this experiment, students learn a wide range of different topics from biological fundamentals (blood pressure) to sensor basics (bridge circuits, strain gauge, pressure sensor) to automated evaluation of measurements in technical systems. Apart from function principles they also learn practical application as well as problems in using sensors, e.g. the need for correct calibration. During the experiment, VenDASys helps students with addressing the different stages, simplifying the electronics and allowing students and teachers to focus on the experiment. At the same time, they learn and understand the principles behind an everyday object like an automatic BP monitor thus taking the technology out of its "black box". Finally, and for our purpose perhaps most important, students are shown that science and technology is fun and that they themselves can realize similar experiments with a minimum of effort, especially using VenDASys as a versatile platform.

\section{Evaluation of the experiment with high school students}

After the experiment, the participating students were asked to evaluate the experiment in several categories such as the experiment was fun using a five point grade scale ranging from I totally agree to I totally disagree and also the experiment overall on a scale from excellent to awful. Fig. 12 shows results of this survey for the first time this experiment was used in our learning lab SinnTec on March 25, 2009, by 10 students.

While the number of students is of course too small for a sound statistical analysis the survey results and also comments by the students are encouraging. While the overall assessment is similar (slightly better) compared to other SinnTec experiments, the agreement in the category the experiment was fun is exceptionally high at $90 \%$.

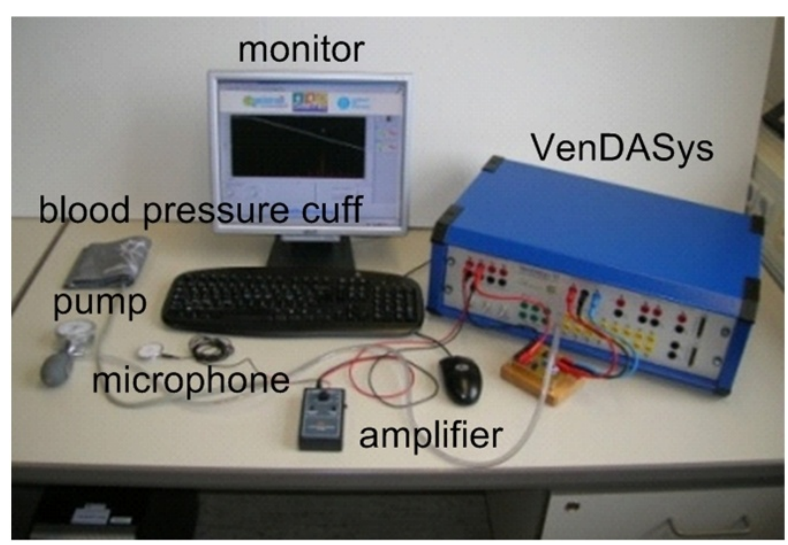

Figure 10. Complete experimental set-up for blood pressure monitoring.

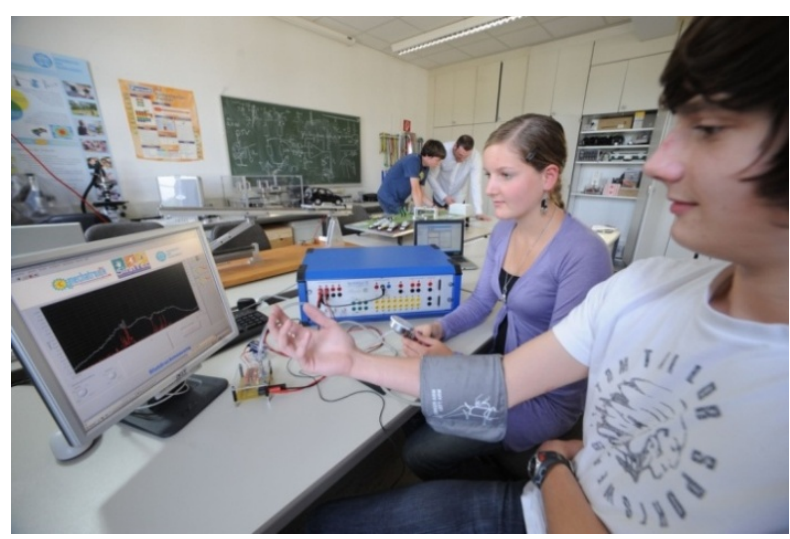

Figure 11. Students measuring their BP after setting up the experiment.
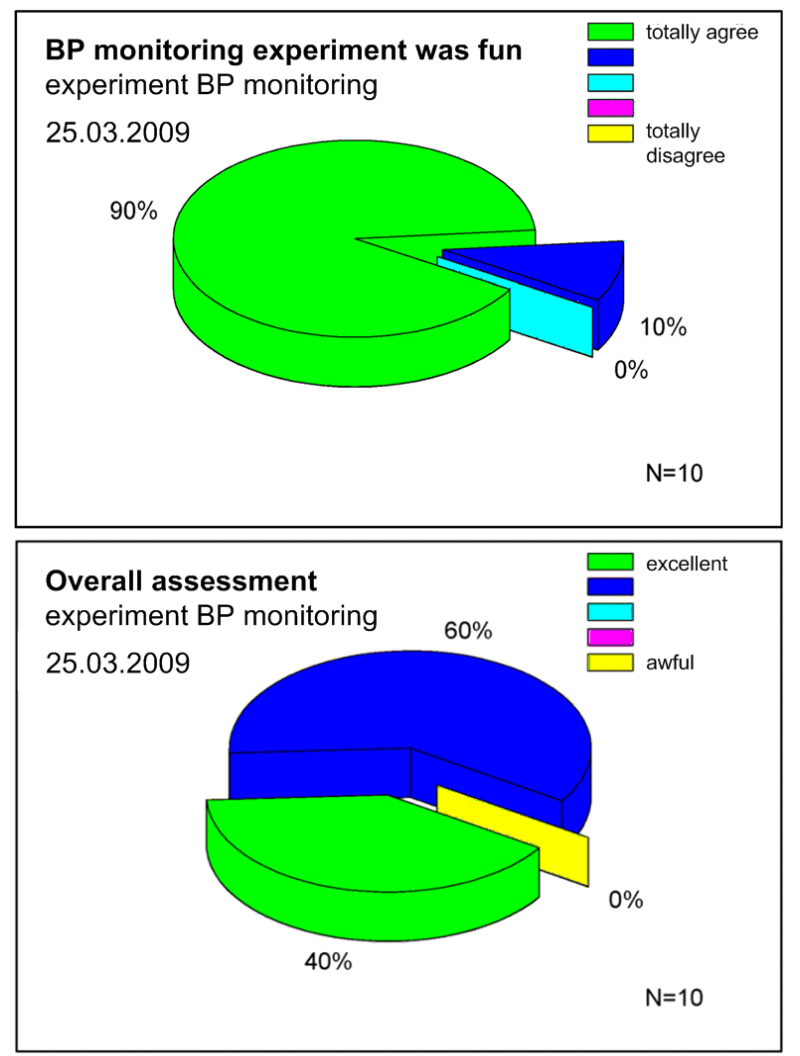

Figure 12. Exemplary evaluation results of the experiment blood pressure monitoring by participating students, 25.03.2009 
We attribute the higher fun factor of the experiment to two things: (1) the topic with its medical background and every day applicability was probably more attractive for students; (2) the VenDASys, which was used for the first time in a complex experimental setting, allowed students to concentrate more on the experiment and its implications than in other examples, where more effort and focus was required for the required electronics.

\section{FURTHER EXPERIMENTS}

Besides the BP monitoring experiment described in detail above, we are currently developing further experiments, each of which addresses another sensor principle and an application example taken from every day experience. One experiment, implemented by a high school student in an extracurricular project, addresses the automatic identification of different solvents with a gas sensor. The experiment simulates a freight depot, where model carriages are shunted to their respective tracks. Here, VenDASys is used to automate the sequential control as well as the selection of the correct track (Fig. 13).

\section{SELF-STUDY COURSES}

In addition to these experiments, we are also developing a self-study course on electronics. The aim of selflearning courses for electronics is to impart basic knowledge of electronics to high school students through an intuitive method of teaching. The primary aim is to achieve a deeper insight by active participation of the students through their own measurements. The emphasis on the mathematical context, often being a stumbling block for many students, will be reduced. Instead, the graphical presentation, fundamental understanding and verbal explanations will be accentuated [12].

The VenDASys platform offers all the necessary measuring instruments, power supplies as well as voltage and current sources. Coupled with the software LabVIEW, the industry standard in the field of measurement and control, this system will enable a variety of applications, starting from a system self test and characterization of simple electronic components which the pupils can also develop and realize themselves. This will lead to gradual learning and the ability to build more complex applications as shown in Fig. 14.

An important aspect of this self-study course is the realization of html-based the course material. Pupils and high school students are already well familiar with the operation of web browsers but have rarely used this knowledge in connection with a physics lab or other school experiments. We therefore expect a higher motivation of the students. In addition and in contrast to regular course materials on paper, videos can be added which can be played as often as necessary to illustrate important aspects. Furthermore, we can also create links to the required basics and learning abilities for each new chapter, so that a search for the right places in the script is no longer necessary. Since large texts are often very deterrent, explanations can be integrated with the experiments without too many words on video.

The course will consist of two parts:

1. Pupils and high school students from grade 7 , who at least know the concepts of current, voltage and resistance including Ohms law will learn to measure voltage, current and resistance, parallel and series circuits as well as voltage dividers etc. Based on these fundamentals, the course will guide them through the development of electronic thermometer based on a Pt1000 temperature sensor.

2. Students from higher grade levels (or those who have completed the first course) will receive an introduction to semiconductor electronics. It should comprise, e.g. characteristic curves of diodes and transistors to be determined with the VenDASys using programmed voltage or current sources. Afterwards the pupils have the opportunity to address various projects, to pick an experiment and implement it themselves based on the acquired knowledge.

To complement the electronics self-study course, a second course with an introduction to LabVIEW will be implemented also in html-format with many demonstration videos and screen sequences. This gives the students the opportunity to realize more complex project ideas based on hard- and software plus the possibility participate in competitions for junior researchers, e.g. "Jugend forscht” or comparable international competitions.

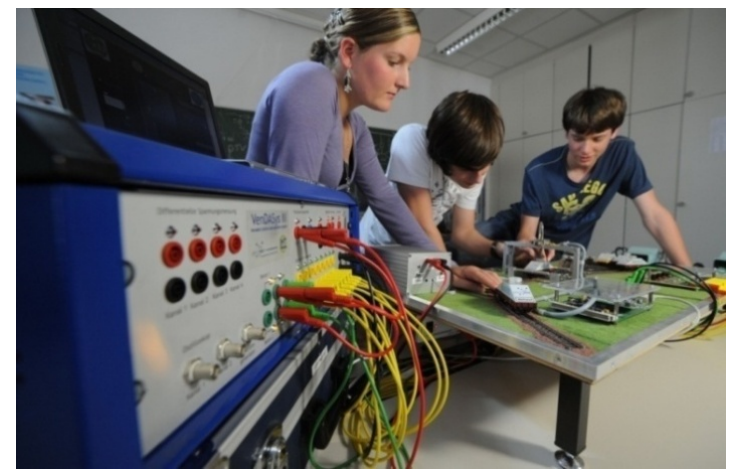

Figure 13. Experiment freight depot with automatic carriage identification

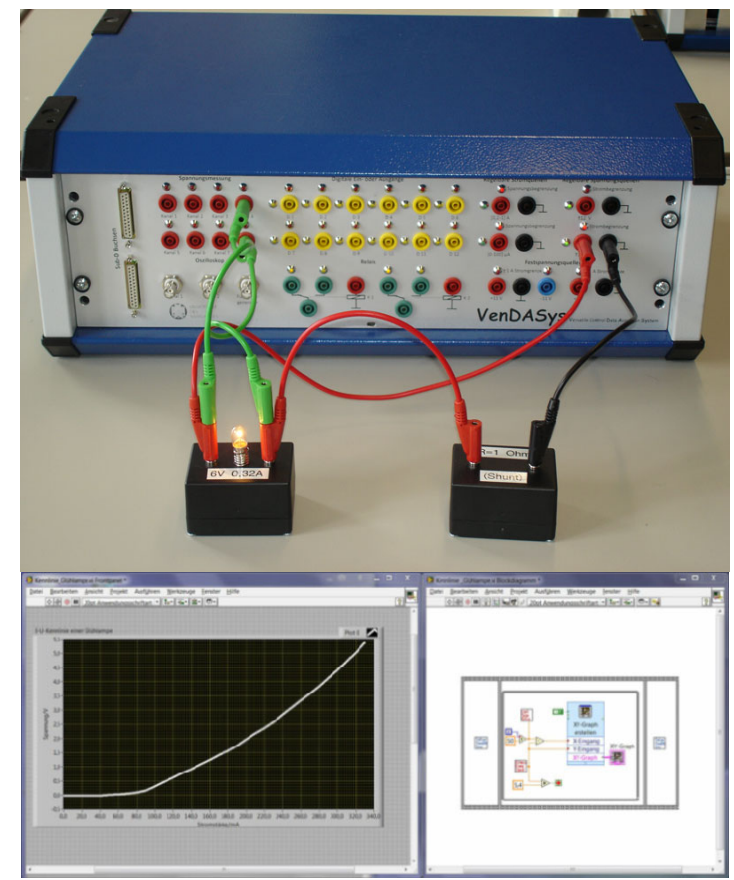

Figure 14. Exemplary realization of an experiment with the VenDASys platform in the self-study course: the U-I-characteristic of a light bulb is measured showing the change of resistance caused by heating the

filament. Top: experimental setup, bottom: resulting characteristic curve (left) measured automatically using a simple LabVIEW-VI (right). 


\section{CONCLUSION AND OUTLOOK}

With the approach described in detail above the following educational aspects can be addressed:

VenDASys is based on a strictly modular, open approach, i.e. all hardware interfaces as well as the software codes are fully available for every user. This enables schools and universities to interface existing and to add new experiments simply and cost efficiently - a crucial aspect for most institutions.

During the development and later in the dissemination of the course and the different experiments we are cooperating closely with different school teachers and their students to receive feedback and input. In addition, the input by teachers will be used to ensure that the VenDASysGUIs fulfill strict didactic requirements to allow intuitive use by students and teachers alike. In the first experiments implemented jointly with teachers a didactic scheme will be designed to be used henceforth.

Synergy effects are created by exchanging experiments via our web-site [11]: mechanical designs, interface electronics and software will be made available for download allowing fast growth of the common experimental pool. Before uploading any element it is checked and, if needed, software is adapted to fit into the didactic scheme allowing intuitive use. In addition, the website will provide user support, e.g. with discussion forum and an FAQ (Frequently Asked Questions) section.

While microsensors and MEMS are our primary concern, we want to expand the application of VenDASys to other fields in engineering and science to make full use of its potential. Besides biology/medicine, as demonstrated with the BP experiment, we think that also chemistry provides high potential for VenDASys application, for example monitoring of reactions or even experiments on bio-fuel generation.

Currently, the system is extensively tested together with pilot users, the results will be continuously reviewed for further improvement. It is clearly not our goal to compete with commercial products offering highly sophisticated systems specifically for the classroom. Instead, our focus is on a system which can be used by students individually and in teams when dealing with technical and scientific subjects in general and MEMS topics or sensor applications in particular. To achieve this goal, self-study courses on electronics and LabVIEW programming will be developed and adapted closely to the VenDASys platform.

\section{ACKNOWLEDGMENT}

We thank Richard Baumbach, Wirtschaftsgymnasium Saarbrücken, for his ideas and support in developing the VenDASys concept and in disseminating it to other schools.

\section{REFERENCES}

[1] Proc. IEEE Conference: Meeting the Growing Demand for Engineers and Their Educators 2010-2020, ISBN: 978-1-4244-1916-6.
[2] A. Ferrero et al., "ReMLab: A Java-Based Remote, Didactic Measurement Laboratory”, IEEE Trans. Instr. A. Meas., Vol. 52, No. 3, 2003.

[3] AWNET: Aus- und Weiterbildungsnetzwerke für die Mikrosystemtechnik, www.mst-ausbildung.de

[4] Aus- und Weiterbildungsfoundry für Prozesstechnologien in der MST, www.pro-mst.de

[5] A. Schütze, A. Picard, B. Kramer, T. Conrad, "Universities, Research Institutions, Industry \& Schools Can Jointly Increase the Interest of Pupils in Engineering”, in Ref. [1].

[6] T. Conrad, A. Schütze, "A contribution for increasing the interest of high-school students for MEMS technology, engineering, and physics”, Measurement 40, 2007, pp. 224-232. doi:10.1016/ j.measurement.2006.06.022

[7] Image online: http://www.learnsite.ch/naturlehre/7 schuljahr/ blut _und_blutkreislauf/blut/blut_blutdruckmessung/blut-druckmessu ng_rr.png

[8] Wikipedia; http://en.wikipedia.org/wiki/Blood_pressure

[9] Image online: http://de.wikipedia.org/wiki/Datei:Korotkow_deut sch.png

[10] Image online: http://www.kfztech.de/kfztechnik/fahrwerk/reifen/ drucksensor_sensoNor.jpg

[11] http://www.vendasys.uni-saarland.de

[12] R. Sallier, B. Brück: „Einsatz der Experimentierplattform VenDASys am Beispiel eines Elektronik-Selbstlernkurses“, Tagung „Keine Angst vor Wissenschaft“, Praxisbeispiele 6: Spezielle Modelle - innovative Wege, Gießen, Germany, May 28-29, 2010.

\section{AUTHORS}

R. Sallier studied electrical engineering and received his M.Sc. from the technical university Kaiserslautern in 2007. He is currently a research associate at the Lab for Measurement Technology (LMT) in the Department of Mechatronics, Saarland University, Saarbrücken, Germany (e-mail: r.sallier@lmt.uni-saarland.de). His research emphasis is the development of the VenDASys platform and the evaluation of the effects achieved with this platform in schools and student projects.

R. Mißler completed a teachers training program for vocational schools in 2009. In the frame of his scientific thesis project at LMT, he developed the blood pressure monitoring experiment.

B. Brück teaches Physics and Mathematics at the Max Planck Gymnasium, Saarlouis, Germany.

Prof. Dr. A. Schütze studied physics at the RWTH Aachen and received his Ph.D. in applied physics from JLU Gießen in 1994. Since 2000 he is full professor at Saarland University and head of the Laboratory for Measurement Technology (e-mail: schuetze@lmt.unisaarland.de).

This work is supported in part by the Saarland Ministry of Economics and Science. We would like to thank for the financial support.

Submitted: June $10^{\text {th }}, 2010$. Published as resubmitted by the authors July $13^{\text {th }}, 2010$. 